\title{
Theory of slow-light semiconductor optical amplifiers
}

\section{Saldutti, Marco; Rasmussen, Thorsten S.; Gioannini, Mariangela; Mørk, Jesper}

\section{Published in:}

Optics Letters

Link to article, DOI:

10.1364/OL.403446

Publication date:

2020

Document Version

Early version, also known as pre-print

Link back to DTU Orbit

Citation (APA):

Saldutti, M., Rasmussen, T. S., Gioannini, M., \& Mørk, J. (2020). Theory of slow-light semiconductor optical amplifiers. Optics Letters, 45(21), 6022-6025. https://doi.org/10.1364/OL.403446

\section{General rights}

Copyright and moral rights for the publications made accessible in the public portal are retained by the authors and/or other copyright owners and it is a condition of accessing publications that users recognise and abide by the legal requirements associated with these rights.

- Users may download and print one copy of any publication from the public portal for the purpose of private study or research.

- You may not further distribute the material or use it for any profit-making activity or commercial gain

- You may freely distribute the URL identifying the publication in the public portal

If you believe that this document breaches copyright please contact us providing details, and we will remove access to the work immediately and investigate your claim. 


\title{
Theory of slow-light semiconductor optical amplifiers
}

\author{
Marco Saldutti ${ }^{1,2,},{ }^{,}$Thorsten S. Rasmussen ${ }^{2}$, Mariangela Gioannini ${ }^{1}$, And Jesper Mørk ${ }^{2}$ \\ ${ }^{1}$ Department of Electronics and Telecommunications, Politecnico di Torino, IT-10129 Turin, Italy \\ ${ }^{2}$ DTU Fotonik, Technical University of Denmark, DK-2800 Kongens Lyngby, Denmark \\ *Corresponding author: marco.saldutti@polito.it
}

Compiled October 18, 2020

We have developed an efficient framework for analyzing the reflection and transmission properties of semiconductor photonic crystal optical amplifiers. Specifically, we have investigated the use of slow-light to enhance the gain of short integrated amplifiers. We find that the expected enhancement in transmission is limited by distributed feedback induced by the material gain itself. Such back-scattering is further enhanced by the refractive index variation associated with the linewidth enhancement factor. The inclusion of this effect reveals that for a given material gain, devices with smaller linewidth enhancement factor may offer better performance.

(C) 2020 Optical Society of America

http://dx.doi.org/10.1364/ao.XX.XXXXXX

Enhancing the modal gain coefficient [1] is one of the multiple possibilities offered by slow-light (SL) in photonic crystal $(\mathrm{PhC})$ structures [2]. Experimentally verified [3], this gain enhancement enables the realization of shorter optical amplifiers with promising applications to photonic integrated circuits [4], as well as shorter lasers [5]. However, a fundamental limitation to this approach is imposed by the gain itself [6]. SL is also known to enhance the impact of refractive index variations. In a $\mathrm{PhC}$ waveguide, a uniform refractive index variation results in a band edge shift, with interesting reported applications $[7,8]$.

In this letter, we perform a detailed investigation of the impact of SL on the optical propagation in a PhC semiconductor optical amplifier (SOA). Our analysis is restricted to the linear regime, i.e. sub-milliwatt power levels [9], appropriate for onchip interconnects.. Compared to previous works $[9,10]$, we utilize an approach adapted from theory of distributed feedback (DFB) lasers to offer new insights. Specifically, we consider the practical case of a buried heterostructure $(\mathrm{BH})$ active region [11] and account for the refractive index variation due to the active material linewidth enhancement factor (LEF). The presence of material gain induces coupling between the Bloch modes of the amplifier, which are otherwise uncoupled; consequently, a strong reflection builds up, which limits the achievable gain. If the LEF is accounted for, this degrading phenomenon may occur at smaller gain values and/or at wavelengths farther from the band edge as compared to the case when the LEF is neglected, an effect which was not previously considered.

In the spirit of coupled-mode theory (CMT), the electromagnetic state of a PhC line-defect waveguide with a complex refractive index can be represented as [12]

$$
\left[\begin{array}{l}
\mathbf{E}(\mathbf{r}) \\
\mathbf{H}(\mathbf{r})
\end{array}\right]=c_{+}(z)\left[\begin{array}{l}
\mathbf{e}_{+}(\mathbf{r}) \\
\mathbf{h}_{+}(\mathbf{r})
\end{array}\right]+c_{-}(z)\left[\begin{array}{l}
\mathbf{e}_{-}(\mathbf{r}) \\
\mathbf{h}_{-}(\mathbf{r})
\end{array}\right]
$$

In this formulation, the material gain (or absorption) induced by carrier density is viewed as a perturbation to an unperturbed waveguide with purely real refractive index. In Eq. (1), $z$ is the propagation coordinate and $\mathbf{r}$ the position vector. $\mathbf{e}_{+}\left(\mathbf{e}_{-}\right)$is the forward-propagating (backward-propagating) electric field of the guided Bloch mode at the angular frequency $\omega$; similarly, $\mathbf{h}_{ \pm}$ are the magnetic fields. These modes are normalized according to the power flux which they carry [12]. Moreover, they are $z$-periodic, with the period given by lattice constant $a$. The amplitudes $c_{ \pm}$generally depend on the perturbation, as well as on $z$ and $\omega$. They are given by $c_{ \pm}=\psi_{ \pm} e^{ \pm i k_{z} z}$, where $k_{z}$ is the (real) wavenumber of the unperturbed waveguide, while $\psi_{ \pm}$ are slowly-varying spatial envelopes. If nonlinear effects are neglected, the envelopes are governed by two coupled differential equations [13]

$$
\begin{aligned}
\partial_{z} \psi_{+} & =i \kappa_{\mathrm{FF}} \psi_{+}+i \kappa_{\mathrm{FB}} e^{+i 2 \delta z} \psi_{-} \\
-\partial_{z} \psi_{-} & =i \kappa_{\mathrm{BF}} e^{-i 2 \delta z} \psi_{+}+i \kappa_{\mathrm{FF}} \psi_{-}
\end{aligned}
$$

which we call coupled-Bloch-mode (CBM) equations. Here, $\delta=\pi / a-k_{z}$ is the detuning from the band edge. In contrast to the classical CMT of DFB lasers [14], the self- $\left(\kappa_{\mathrm{FF}}\right)$ and cross-coupling coefficients $\left(\kappa_{\mathrm{FB}}\right.$ and $\left.\kappa_{\mathrm{BF}}\right)$ strongly depend on frequency. They are given by $\kappa_{x} \approx(\omega / c)\left(\Delta n_{r}+i n_{i}\right) S(\omega) \Gamma_{x}(\omega)$, with $x=\mathrm{FF}, \mathrm{FB}, \mathrm{BF}$. Here, $c$ is the vacuum light speed and $S=n_{g}(\omega) / n_{\text {slab }}$ the slow-down factor, with $n_{g}$ and $n_{\text {slab }}$ being, respectively, the group index and (real) slab refractive index of the unperturbed waveguide (material dispersion is neglected). $n_{i}$ is the imaginary refractive index associated with carrier density and $\Delta n_{r}$ a possible, real variation to $n_{\text {slab }}$ (not necessarily due to carrier density). $\Gamma_{\mathrm{FF}}$ and $\Gamma_{\mathrm{FB}}\left(\Gamma_{\mathrm{BF}}=\Gamma_{\mathrm{FB}}^{\star}\right)$ are coefficients depending on the spatial overlap between the perturbation and Bloch modes of the unperturbed waveguide. They are given, respectively, by $\Gamma_{\mathrm{FF}}=(1 / a) \int_{a} \Gamma_{11}(z) d z$ and $\Gamma_{\mathrm{FB}}=(1 / a) \int_{a} \Gamma_{12}(z) e^{+i \frac{2 \pi}{a}} z d$, where

$$
\Gamma_{11}(z)=\frac{a \int_{A} \epsilon_{0} n_{\text {slab }}^{2}\left|\mathbf{e}_{+}(\mathbf{r})\right|^{2} F(\mathbf{r}) d A}{\int_{V}\left[\epsilon_{0} n_{b}^{2}(\mathbf{r})\left|\mathbf{e}_{+}(\mathbf{r})\right|^{2}\right] d V}
$$




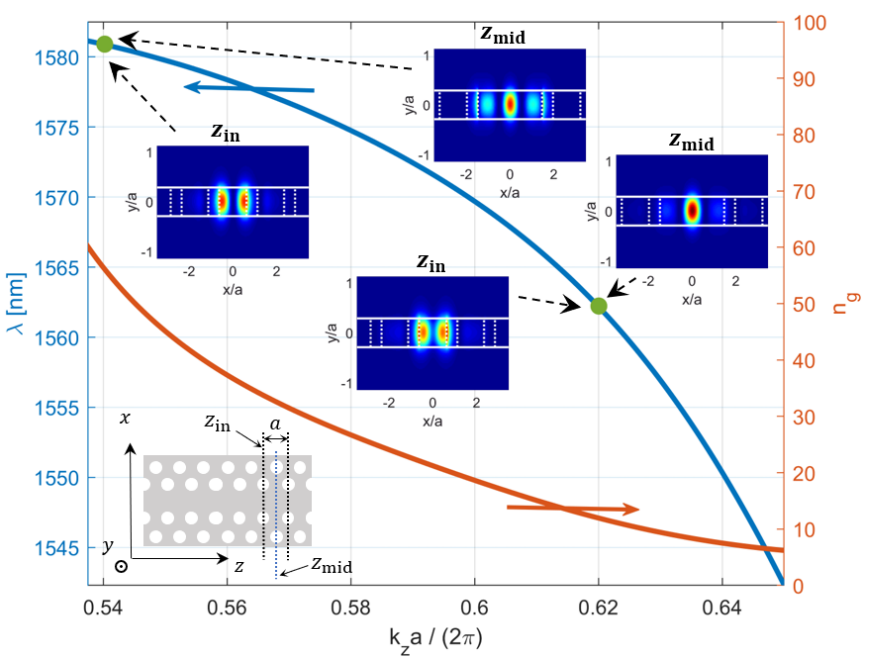

Fig. 1. Dispersion curve (left) and group index (right) of the fundamental TE-like mode of the unperturbed waveguide. The bottom left inset shows the top view of the unperturbed waveguide and reference planes of a unit cell along the propagation direction. The other insets display the electric field intensity $\left|\mathbf{e}_{+}\right|^{2}$ on the waveguide cross-section at the input $\left(z_{\text {in }}\right)$ and center $\left(z_{\text {mid }}\right)$ of a unit cell at $k_{z} a /(2 \pi)=0.54\left(n_{g} \approx 56\right)$ and $k_{z} a /(2 \pi)=0.62\left(n_{g} \approx 12\right)$. The vertical (horizontal) white lines delimit the air holes (semiconductor slab).

$$
\Gamma_{12}(z)=\frac{a \int_{A} \epsilon_{0} n_{\mathrm{slab}}^{2}\left[\mathbf{e}_{-}(\mathbf{r}) \cdot \mathbf{e}_{+}^{*}(\mathbf{r})\right] F(\mathbf{r}) d A}{\int_{V}\left[\epsilon_{0} n_{b}^{2}(\mathbf{r})\left|\mathbf{e}_{+}(\mathbf{r})\right|^{2}\right] d V}
$$

$\Gamma_{11}$ and $\Gamma_{12}$ are normalized coupling coefficients derived in [12]. $V$ is the volume of a supercell, $A$ the transverse cross-section at $z$ and $n_{b}=n_{\text {slab }}\left(n_{b}=n_{\text {clad }}\right)$ in the slab (cladding) is the background refractive index. $F$ is the distribution function of the perturbation, represented by the active layers of quantum wells (QWs) or quantum dots (QDs), which are assumed to undergo spatially uniform pumping. They may uniformly extend across the lateral direction (in what we call an all-active waveguide) [3] or be confined to the line-defect, such as for waveguides with a $\mathrm{BH}$ active region. In either case, $\Gamma_{11}$ and $\Gamma_{12}$ are $z$-periodic, with period $a$. Therefore, they may be expanded in a Fourier series. In this case, one finds $\Gamma_{\mathrm{FF}}\left(\Gamma_{\mathrm{FB}}\right)$ to be the dominant harmonic in the expansion of $\Gamma_{11}\left(\Gamma_{12}\right)$, thus obtaining Eqs. (2a)-(2b) from those derived in [12]. This is explained in detail in [13] for all-active waveguides; we have found that the same holds in the case of a $\mathrm{BH}$ active region. $\Gamma_{\mathrm{FF}}$ and $\Gamma_{\mathrm{FB}}$ depend, in principle, on the number and position of the active layers along the direction orthogonal to the slab $(y-)$. However, the field confinement along the $y$-direction is due to total internal reflection. Owing to the high refractive index contrast between the air and slab material, this is weakly dependent on $\omega$ and $z$ as compared to confinement along the lateral direction $(x-)$, which is due to the photonic band gap. Consequently, we may evaluate $\Gamma_{\mathrm{FF}}$ and $\Gamma_{\mathrm{FB}}$ by assuming the perturbation to be homogeneous within the slab along the $y$-direction. Under these conditions, $n_{i}$ reflects the modal gain coefficient $g_{0}$, with $n_{i}=-(c / \omega) g_{0} / 2$ and $g_{0}=\Gamma_{y} g_{\text {mat }}$. Here, $g_{\text {mat }}$ is the material gain and $\Gamma_{y}$ the optical confinement factor along the $y$-direction within the active layers (assumed to be frequency-independent).

In the following, we assume $n_{\text {clad }}=1$, corresponding to the slab being suspended in air [11], and $n_{\text {slab }}=3.17$, representative

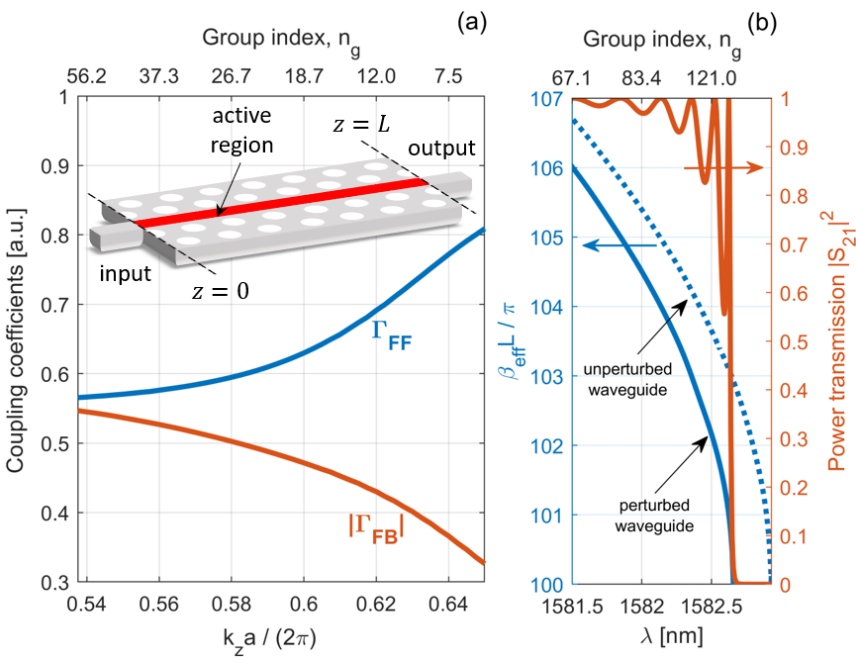

Fig. 2. (a) $\Gamma_{\mathrm{FF}}$ and $\Gamma_{\mathrm{FB}}$ for a waveguide with a $\mathrm{BH}$ active region. The inset illustrates the device under study in this letter. (b) Impact of a given $\Delta n_{r}$ on the optical propagation in a waveguide with $n_{i}=0$ and $L=100 a$. The left axis shows the effective propagation constant of the perturbed waveguide with $\Delta n_{r}=-0.001$ (solid) normalized to $\pi / L$. The dispersion curve of the unperturbed waveguide is also shown (dotted). The perturbation is limited to the line-defect. The right axis displays the power transmission through the perturbed waveguide.

of the InP platform. The slab thickness is $250 \mathrm{~nm}$ and the hole radius $0.25 a$, with $a=438 \mathrm{~nm}$. Fig. 1 illustrates the dispersion relation (left axis) and group index (right axis) of the unperturbed waveguide, computed by the plane wave eigensolver MIT Photonic-Bands [15]. The top view of the unperturbed waveguide is displayed by the bottom left inset. The other insets show the electric field intensity $\left|\mathbf{e}_{+}\right|^{2}$ on the waveguide cross-section at the input $\left(z_{\text {in }}\right)$ and center $\left(z_{\text {mid }}\right)$ of a unit cell for two different wavelengths $(\lambda)$, corresponding to moderately low $\left(n_{g} \approx 12\right)$ and high $\left(n_{g} \approx 56\right)$ group index. As the group index increases, the mode spreads in the lateral direction [16]. Consequently, if the active region is limited to the line-defect, a gradual decrease in $\Gamma_{\mathrm{FF}}$ is to be expected. This is evidenced by Fig. 2(a), showing $\Gamma_{\mathrm{FF}}$ for a waveguide with a $\mathrm{BH}$ active region. In addition, here we also show that a strong cross-coupling (i.e. $\Gamma_{\mathrm{FB}}$ ) builds up while approaching the band edge, as explained in detail in [13] for all-active waveguides. The same dependence is found here for a $\mathrm{BH}$ active region. For the choice of unit cell in Fig. 1, $\Gamma_{\mathrm{FB}}$ turns out to be (to very good approximation) real and positive. To easily incorporate the LEF, we assume a linear dependence on carrier density of the material gain and real part of the perturbed waveguide refractive index, $n_{r}$. By introducing the LEF, $\alpha_{H}=-\left(d n_{r} / d N\right) /\left(d n_{i} / d N\right)$ [17], with $N$ being the carrier density, and assuming $n_{\text {slab }}$ to coincide with $n_{r}$ at the transparency carrier density, we may then reformulate $n_{r}$ as $n_{r}(N)=n_{\text {slab }}+\alpha_{H} n_{i}(N)$, leading to $\Delta n_{r}=\alpha_{H} n_{i}$. Finally, the scattering loss is modeled with a phenomenological approach by adding a loss contribution to the self-coupling coefficient, i.e. $\kappa_{\mathrm{FF}}=(\omega / c)\left(\Delta n_{r}+i n_{i}\right) S \Gamma_{\mathrm{FF}}+i\left[\alpha_{1} S+\alpha_{2} S^{2}\right] / 2$, where, $\alpha_{1}$ and $\alpha_{2}$ account, respectively, for disorder-induced coupling with radiation modes and back-scattering [18]. We also note that the CBM equations are cast in a form that is suitable for being combined with a more rigorous treatment of disorder $[5,19]$. 

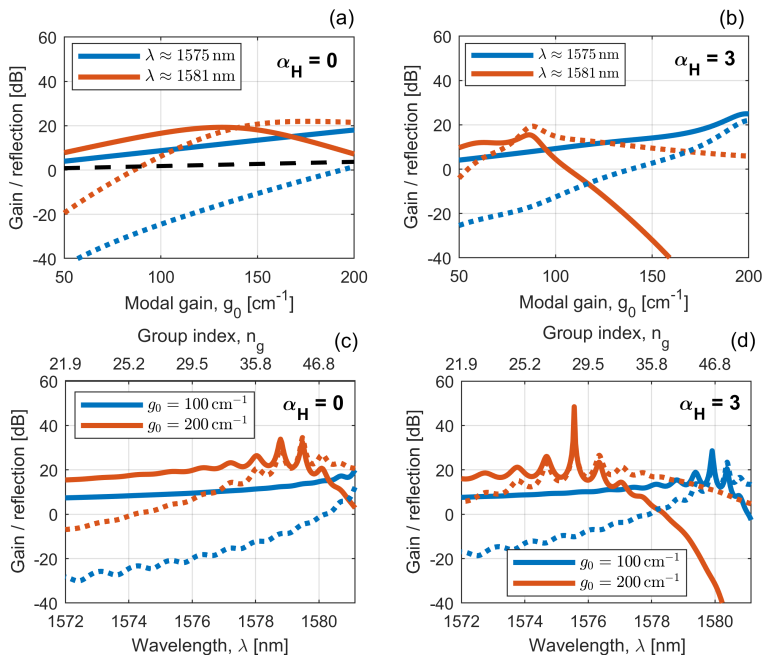

Fig. 3. Amplifier gain (solid) and reflection (dotted) with (a, c) $\alpha_{H}=0$ and $(b, d) \alpha_{H}=3$. They are plotted: $(a, b)$ versus the modal gain, $g_{0}$, at $\lambda \approx 1575 \mathrm{~nm}$ (blue) and $\lambda \approx 1581 \mathrm{~nm}$ (red); (c, d) versus wavelength, $\lambda$, at $g_{0}=100 \mathrm{~cm}^{-1}$ (blue) and $g_{0}=200 \mathrm{~cm}^{-1}$ (red). The dashed, black line in (a) is the gain with SL effects being neglected.

By solving the CBM equations as an initial value problem, the spatial dependence of the fields may be written as

$$
\left[\begin{array}{c}
\mathbf{E}(\mathbf{r}) \\
\mathbf{H}(\mathbf{r})
\end{array}\right]=\tilde{F}\left[\begin{array}{c}
\tilde{\mathbf{e}}_{+}(\mathbf{r}) \\
\tilde{\mathbf{h}}_{+}(\mathbf{r})
\end{array}\right] e^{+i \tilde{k}_{z} z}+\tilde{B}\left[\begin{array}{c}
\tilde{\mathbf{e}}_{-}(\mathbf{r}) \\
\tilde{\mathbf{h}}_{-}(\mathbf{r})
\end{array}\right] e^{-i \tilde{k}_{z} z}
$$

Here, $\tilde{\mathbf{e}}_{ \pm}=\mathbf{e}_{ \pm}+r_{ \pm} e^{\mp i \frac{2 \pi}{a} z} \mathbf{e}_{\mp}$ is the electric field of the forward(+) and backward-propagating (-) Bloch mode of the perturbed waveguide, with analogous expressions for the magnetic fields $\tilde{\mathbf{h}}_{ \pm}$. Each Bloch mode of the perturbed waveguide results from the interference of the forward- and backward-propagating Bloch modes of the unperturbed waveguide, with $r_{+}$and $r_{-}$ determining the strength of the backward component as compared to the forward one and viceversa. $\tilde{F}$ and $\tilde{B}$ are the amplitudes, while $\tilde{k}_{z}=\beta_{\text {eff }}-i g_{\text {eff }} / 2$ is the (complex) wavenumber. The effective propagation constant and net modal gain are, respectively, $\beta_{\text {eff }}=\operatorname{Re}\left\{\lambda_{1}\right\}+\pi / a$ and $g_{\text {eff }}=-2 \operatorname{Im}\left\{\lambda_{1}\right\}$, with $\lambda_{1}= \pm \sqrt{\left(\kappa_{\mathrm{FF}}-\delta\right)^{2}-\kappa_{\mathrm{FB}} \kappa_{\mathrm{BF}}}$. In the presence of gain, the sign of the square root is selected such that $\operatorname{Im}\left\{\lambda_{1}\right\}$ and $\operatorname{Im}\left\{\kappa_{\mathrm{FF}}\right\}$ have the same sign. Finally, $r_{ \pm}=-\kappa_{\mathrm{BF} ; \mathrm{FB}} /\left[\left(\kappa_{\mathrm{FF}}-\delta\right)+\lambda_{1}\right]$. This approach is similar to that suggested in [20] for DFB lasers. Without cross-coupling, one finds $\beta_{\text {eff }}=k_{z}-S \Gamma_{\mathrm{FF}} g_{0} \alpha_{H} / 2$ and $g_{\text {eff }}=S \Gamma_{\mathrm{FF}} g_{0}-\left(\alpha_{1} S+\alpha_{2} S^{2}\right)$. Therefore, a simple enhancement of the modal gain proportional to the slow-down factor may be only expected in the absence of cross-coupling, which is the case when we operate far from the band edge. Through the same procedure leading to Eq. (4), one may derive the scattering matrix $\underline{\underline{S}}$ relating the amplitudes of the Bloch modes exiting and entering the perturbed waveguide, i.e. $\left[c_{-}(0) c_{+}(L)\right]^{T}=$ $\underline{S}\left[c_{+}(0) c_{-}(L)\right]^{T}$, with $T$ denoting the transpose operator. Here, $\bar{z}=0(z=L)$ corresponds to the input (output) of the active region (see the inset of Fig. 2(a)). The elements of $\underline{\underline{S}}$ are given by

$$
S_{11 ; 22}=\frac{-\left(e^{+2 i \lambda_{1} L}-1\right) r_{ \pm}}{1-r_{+} r_{-} e^{+2 i \lambda_{1} L}} e^{\mp i \frac{2 \pi}{a} L}
$$
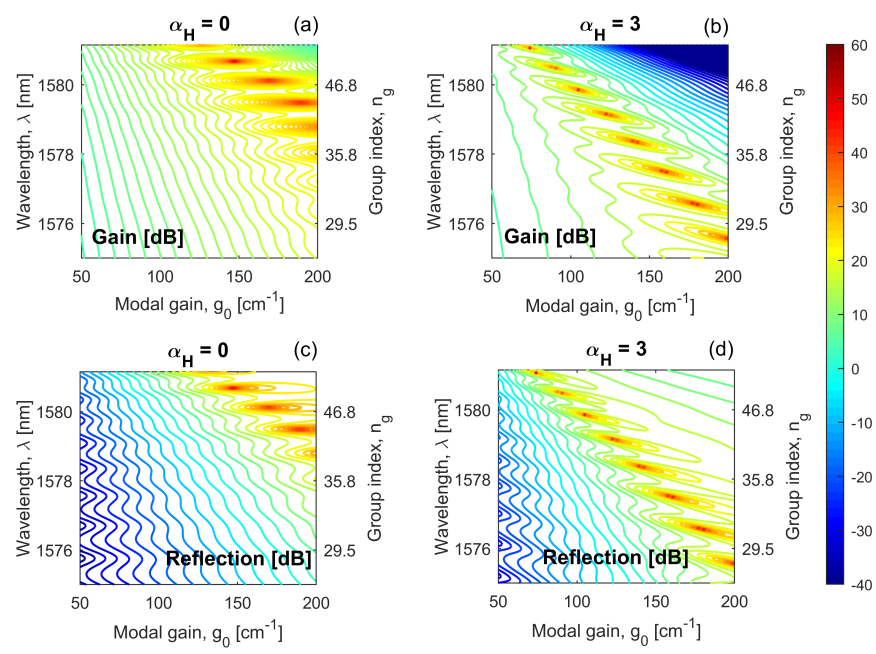

Fig. 4. (a, b) Amplifier gain and (c, d) reflection with (a, c) $\alpha_{H}=0$ and $(b, d) \alpha_{H}=3$ versus wavelength, $\lambda$, and modal gain, $g_{0}$.

$$
S_{12 ; 21}=\frac{\left(1-r_{+} r_{-}\right) e^{+i \lambda_{1} L}}{1-r_{+} r_{-} e^{+2 i \lambda_{1} L}} e^{+i \frac{\pi}{a} L}
$$

Here, $\left|S_{21}\right|^{2}\left(\left|S_{11}\right|^{2}\right)$ is the normalized power flux transmitted (backscattered) by the perturbed waveguide in the ideal case of continuity boundary conditions at the input and output interfaces, thus describing an intrinsic effect of the amplifier.

For the sake of clarity, we first consider the case of a sole refractive index variation $\Delta n_{r}$. For this purpose, Fig. 2(b) shows the power transmission $\left|S_{21}\right|^{2}$ through a waveguide with $\Delta n_{r}=$ $-0.001, n_{i}=0, \alpha_{1}=\alpha_{2}=0$ and $L=100 a$ (right axis) computed by Eq. (5b). Since there is neither gain, nor loss, one finds $\left|S_{11}\right|^{2}=1-\left|S_{21}\right|^{2}$. The squared magnitude of $S_{11}$ represents the fraction of power backscattered at the interface between two adjacent sections of a PhC waveguide only differing (by $\Delta n_{r}$ ) in the slab refractive index, with the second section being the perturbed one and having length $L$. The left axis shows the effective propagation constant of the perturbed waveguide (solid) and that of the unperturbed waveguide (dotted) normalized to $\pi / L$. Due to $\Delta n_{r}$, the band edge of the unperturbed waveguide shifts to shorter wavelengths. This shift cannot be correctly reproduced if the cross-coupling coefficients are neglected [13]. In the induced stop band, the power transmission is zero, as the Bloch modes are evanescent (i.e. $\lambda_{1}$ is purely imaginary, with $g_{\text {eff }}<0$ ) and the waveguide is sufficiently long. Just outside the stop band, the power transmission shows some oscillations, with complete transmission being achieved at wavelengths for which $\beta_{\text {eff }} L / \pi$ is an integer.

We shall now consider the impact of the active material. Due to the adverse effect of disorder on practical devices, we limit the discussion to modes with group index up to around 60 [21] and assume $\alpha_{1}=5 \mathrm{~cm}^{-1}$. For simplicity, we also assume $\alpha_{2}=0$, since this effect only gives rise to a minor, quantitative change of the results for realistic [5] values of $\alpha_{2}$. Furthermore, we assume $L=100 a$ and consider $g_{0}$ values not larger than $200 \mathrm{~cm}^{-1}$. As an example, with typical values of $\Gamma_{y} \approx 2 \%$ per active layer and 5 active layers, this translates into a maximum material gain of $2000 \mathrm{~cm}^{-1}$, which is reasonable [17]. Fig. 3 shows the amplifier gain $\left(\left|S_{21}\right|^{2}\right)$ and reflection $\left(\left|S_{11}\right|^{2}\right)$ as a function of (a, b) modal gain, $g_{0}$, and $(\mathrm{c}, \mathrm{d})$ wavelength, $\lambda$, with $(\mathrm{a}, \mathrm{c}) \alpha_{H}=0$ 

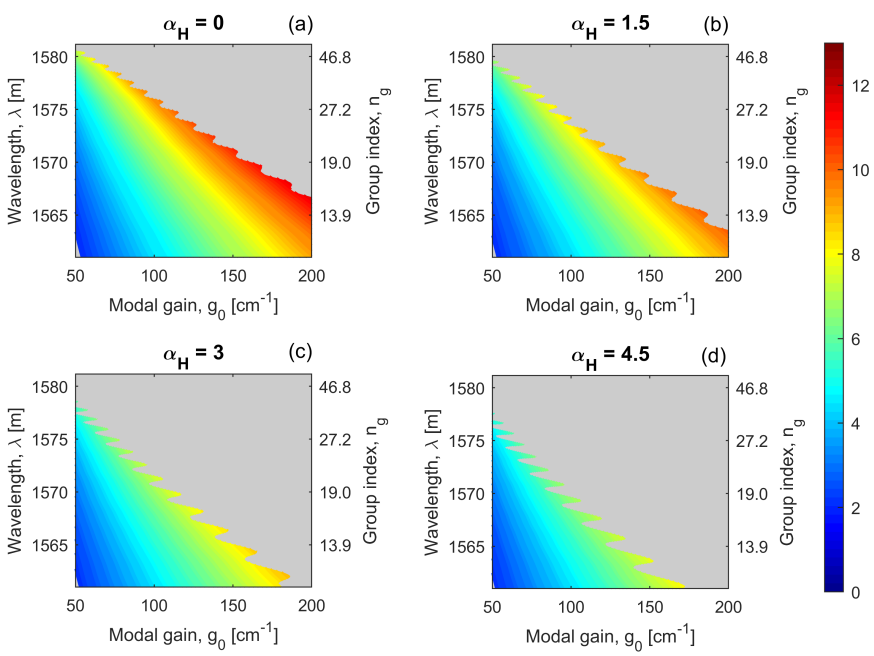

Fig. 5. Amplifier gain in dB with (a) $\alpha_{H}=0$, (b) $\alpha_{H}=1.5$, (c) $\alpha_{H}=3$ and (d) $\alpha_{H}=4.5$. The colored area corresponds to the reflection being smaller than $-20 \mathrm{~dB}$.

and $(\mathrm{b}, \mathrm{d}) \alpha_{H}=3$. The choice $\alpha_{H}=0$ reflects an ideal QD laser, while $\alpha_{H}=3$ is representative of a generic QW laser. For $\alpha_{H}=0$ (see Fig. 3(a)), at $\lambda \approx 1575 \mathrm{~nm}$ (blue) the amplifier gain monotonically increases with the modal gain, while for $\lambda \approx 1581 \mathrm{~nm}$ (red) it achieves a maximum. The appearance of an optimum is explained as resulting from the build-up of a strong reflection. Indeed, the closer $\lambda$ is to the band edge, the stronger the distributed feedback induced by material gain is, owing to the smaller detuning $\delta$ (see Eqs. (2a)-(2b)) as well as the relative increase of $\Gamma_{\mathrm{FB}}$ as compared to $\Gamma_{\mathrm{FF}}$ (see Fig. 2(a)). As a reference, the amplifier gain with $\mathrm{SL}$ effects being neglected (i.e. $S=1, \Gamma_{\mathrm{FF}}=1$ and $\Gamma_{\mathrm{FB}}=0$ ) is also shown (dashed, black). In a complementary fashion (see Fig. 3(c)), at $g_{0}=100 \mathrm{~cm}^{-1}$ (blue) both amplifier gain and reflection increase as the wavelength approaches the band edge, because the slow-down factor grows. However, at $g_{0}=200 \mathrm{~cm}^{-1}$ (red) the distributed feedback beyond a certain wavelength is large enough to induce a steep decrease in the amplifier gain. For $\alpha_{H}=3$ (see Fig. 3(b)), the band edge shifts to shorter and shorter wavelengths as the modal gain grows, due to the increasing $\Delta n_{r}$ induced by the carrier density. Consequently, the amplifier gain at a given wavelength, in dependence of the modal gain, may be slightly enhanced by $\alpha_{H}$, due the larger effective group index resulting from the band edge shift. However, this enhancement comes at the price of an enhanced reflection, due to the combined effect of gainand index-induced distributed feedback. Therefore, the drop in amplifier gain sets in at smaller modal gain values than for $\alpha_{H}=0$. Similarly (see Fig. 3(d)), it occurs, for a given value of modal gain, at wavelengths more detuned from the band edge.

A complete overview is provided by Fig. 4, reporting contour plots of $(a, b)$ amplifier gain and $(c, d)$ reflection with $(a$, c) $\alpha_{H}=0$ and $(b, d) \alpha_{H}=3$ versus wavelength and modal gain. It is seen that both amplifier gain and reflection diverge for certain combinations of wavelength and modal gain. These points correspond to the poles of the scattering matrix $\underline{\underline{S}}$ (i.e. $r_{+} r_{-} e^{+2 i \lambda_{1} L}=1$ ) and therefore to the onset of lasing, which is genuinely sustained by the Bloch modes gain-induced coupling.

The quantification of a maximum amplifier gain depends on the acceptable level of reflection, which varies with the application. As an example, Fig. 5 illustrates the gain corresponding to the reflection being smaller than $-20 \mathrm{~dB}$. The LEF is (a) $\alpha_{H}=0$, (b) $\alpha_{H}=1.5$, (c) $\alpha_{H}=3$ and (d) $\alpha_{H}=4.5$. As $\alpha_{H}$ grows, one has increasingly less freedom, in terms of $\lambda$ and $g_{0}$, to achieve a certain gain. For instance, a maximum gain larger than $11 \mathrm{~dB}$ can be achieved with $\alpha_{H}=0$, but not greater than $8 \mathrm{~dB}$ with $\alpha_{H}=4.5$. This suggests that for a given material gain, QDs may be more attractive than QWs as active layers.

In conclusion, we have provided a theoretical study of the impact of slow-light on the optical propagation properties of PhC SOAs with a buried heterostructure active region. We have found that the maximum amplifier gain is limited by the buildup of a strong reflection, which increases as the operation wavelength approaches the band edge and/or the material gain grows. We have clarified the impact of the linewidth enhancement factor, which further hampers the achievement of a given gain. This implies that devices with smaller linewidth enhancement factor may offer better performance.

Disclosures. The authors declare no conflicts of interest.

J. Mørk and T. S. Rasmussen acknowledge support from Villum Fonden (grant 8692) and the Danish National Research Council (DNRF 147). M. Saldutti gratefully acknowledges fruitful discussions with Prof. I. Montrosset and Prof. R. Orta.

\section{REFERENCES}

1. J. Mørk and T. R. Nielsen, Opt. Lett. 35, 2834 (2010).

2. T. Baba, Nat. Photonics 2, 465 (2008).

3. S. Ek, P. Lunnemann, Y. Chen, E. Semenova, K. Yvind, and J. Mørk, Nat. Commun. 5, 5039 (2014).

4. S. Matsuo and T. Kakitsuka, Adv. Opt. Photon. 10, 567 (2018).

5. W. Xue, Y. Yu, L. Ottaviano, Y. Chen, E. Semenova, K. Yvind, and J. Mork, Phys. Rev. Lett. 116, 063901 (2016).

6. J. Grgić, J. R. Ott, F. Wang, O. Sigmund, A.-P. Jauho, J. Mørk, and N. A. Mortensen, Phys. Rev. Lett. 108, 183903 (2012).

7. Y. A. Vlasov, M. O'Boyle, H. F. Hamann, and S. J. McNab, Nature 438, 65 (2005).

8. T. Tanabe, M. Notomi, H. Taniyama, and E. Kuramochi, Phys. Rev. Lett. 102, 043907 (2009).

9. Y. Chen and J. Mørk, Opt. Express 21, 29392 (2013).

10. E. Mizuta, H. Watanabe, and T. Baba, Jpn. J. Appl. Phys. 45, 6116 (2006).

11. K. Takeda, T. Sato, A. Shinya, K. Nozaki, W. Kobayashi, H. Taniyama, M. Notomi, K. Hasebe, T. Kakitsuka, and S. Matsuo, Nat. Photonics 7, 569 (2013).

12. Y. Chen, J. R. de Lasson, N. Gregersen, and J. Mørk, Phys. Rev. A 92, 053839 (2015).

13. M. Saldutti, P. Bardella, J. Mørk, and M. Gioannini, IEEE J. Sel. Top. Quantum Electron. 25, 1 (2019).

14. H. Kogelnik and C. V. Shank, J. Appl. Phys. 43, 2327 (1972).

15. S. G. Johnson and J. D. Joannopoulos, Opt. Express 8, 173 (2001).

16. N. C. Panoiu, J. F. McMillan, and C. W. Wong, IEEE J. Sel. Top. Quantum Electron. 16, 257 (2010).

17. L. A. Coldren, S. W. Corzine, and M. Mašanović, Diode Lasers and Photonic Integrated Circuits (John Wiley \& Sons, Inc., 2012), 2nd ed.

18. L. O'Faolain, S. A. Schulz, D. M. Beggs, T. P. White, M. Spasenović, L. Kuipers, F. Morichetti, A. Melloni, S. Mazoyer, J. P. Hugonin, P. Lalanne, and T. F. Krauss, Opt. Express 18, 27627 (2010).

19. S. Mazoyer, J. P. Hugonin, and P. Lalanne, Phys. Rev. Lett. 103, 063903 (2009).

20. T. Suhara, Semiconductor laser fundamentals (Marcel Dekker, Inc, 2004), 1st ed.

21. B. Rigal, K. Joanesarson, A. Lyasota, C. Jarlov, B. Dwir, A. Rudra, I. Kulkova, and E. Kapon, Opt. Express 25, 28908 (2017). 